\title{
OCCURRENCE AND DETECTION OF ESBL AMONG ENTEROBACTERIACEAE IN A TERTIARY CARE CENTER IN TRIVANDRUM
}

Ashish Jitendranath ${ }^{1}$, Ivy Vishwamohanan', Geetha Bhai ${ }^{3}$

1Associate Professor, Department of Microbiology, Sree Gokulam Medical College and Research Foundation, Thiruvananthapuram, Kerala, India.

${ }^{2}$ Assistant Professor, Department of Microbiology, Sree Gokulam Medical College and Research Foundation, Thiruvananthapuram, Kerala, India.

3Professor, Department of Microbiology, Sree Gokulam Medical College and Research Foundation, Thiruvananthapuram, Kerala, India. ABSTRACT

\section{BACKGROUND}

Enterobacteriaceae is the primary pathogen isolated predominantly among gram negatives. The prevalence of multidrug-resistant Enterobacteriaceae has increased continuously over the past few years. Extended-spectrum $\beta$ - lactamases (ESBLs) are of particular concern as the rate of resistance has increased over the years. We wanted to characterise Enterobacteriaceae and antibiotic sensitivity patterns with special reference to detection of ESBL.

\section{MATERIALS AND METHODS}

The present study was conducted in the microbiology laboratory of Sree Gokulam Medical College and Research Foundation over a duration of 3 months from Oct 2015 to Dec 2015. Using standard techniques and CLSI protocol we identified the organisms with antibiotic sensitivity.

\section{RESULTS}

20\% of Enterobacteriaceae were ESBL positive with E coli being the predominant organism.

\section{CONCLUSION}

$20 \%$ ESBL production was less compared to studies from other parts of the country.

\section{KEY WORDS}

Antibiotics, ESBL, E coli

HOW TO CITE THIS ARTICLE: Jitendranath A, Vishwamohanan I, Bhai G. Occurrence and detection of ESBL among enterobacteriaceae in a tertiary care center in Trivandrum. J. Evolution Med. Dent. Sci. 2019;8(12):839-842, D0I: $10.14260 /$ jemds/2019/187

\section{BACKGROUND}

Enterobacteriaceae is the primary pathogen isolated among gram negatives. The prevalence of multidrug-resistant Enterobacteriaceae has increased continuously over the past few years. Extended-spectrum $\beta$ - lactamases (ESBLs) are of particular concern as the rate of resistance increased over the years. ${ }^{(1,2)}$

Misuse of drugs in human beings due to irrational use has contributed to this increase in resistance among bacteria. Recently we have started getting examples of the use of antibiotics in veterinary animals and agriculture and its consequent effects on resistance levels in peoples has also come under scrutiny.(3)

Earlier we used to report ESBL from inpatients with prolonged hospitalization, but now ESBL's were also isolated from long-term care facilities and outpatient clinics, which indicate the presence of ESBL in the community.(4)

'Financial or Other Competing Interest': None.

Submission 12-01-2019, Peer Review 07-03-2019,

Acceptance 13-03-2019, Published 25-03-2019.

Corresponding Author:

Dr. Ashish Jitendranath

Associate Professor,

Department of Microbiology,

Sree Gokulam Medical College and Research Foundation,

Venjaramoodu, Thiruvananthapuram,

Kerala, India.

E-mail:ashishjit11@gmail.com

DOI: $10.14260 /$ jemds $/ 2019 / 187$

\section{(c) (1) $(9)$}

Enterobacteriaceae producing extended-spectrum $\beta$ lactamases (ESBLs) have the ability to hydrolyze penicillins, first-second- and $3^{\text {rd }}$ generation cephalosporins and aztreonam.(5)

A high rate of clinical failure among patients who were infected with ESBL producing bacteria and who received initial antimicrobial therapy, especially cephalosporin has been demonstrated. Therefore, detection of ESBL producing organisms is important to ensure effective therapeutic intervention and optimal clinical outcome.(6)

This study aimed to find out the characterization of Enterobacteriaceae and antibiotic sensitivity patterns with special reference to detection of ESBL producing Enterobacteriaceae in patients in a tertiary care hospital in south Kerala.

\section{MATERIALS AND METHODS}

The present study was conducted in the Microbiology laboratory of Sree Gokulam Medical College and Research Foundation over a duration of 3 months from Oct 2015 to Dec 2015.

\section{Study Design}

It is a descriptive study.

All the samples received in the bacteriology section of the laboratory were inoculated on blood agar, MacConkey agar and incubated at $37^{\circ} \mathrm{C}$ for $48 \mathrm{~h}$ before being reported as sterile. The isolates were identified based on Colony 
characteristics, Gram staining, Motility and Conventional biochemical test like oxidase test, catalase test, indole, methyl red, Voges-Proskauer, citrate utilization, urease production oxidative fermentative test (Hugh-Leifson medium) for glucose, utilization of $10 \%$ lactose, gelatin liquefaction, lysine and ornithine decarboxylation, arginine dihydrolase test, growth at $42^{\circ} \mathrm{C}$ and $44^{\circ} \mathrm{C}$, esculin hydrolysis and ONPG test. $(7,8)$

The sensitivity test was performed by Kirby Bauer disc diffusion method using commercially available discs (HiMedia).

The results were interpreted as per the CLSI guidelines1. Extended Spectrum $\beta$-Lactamases (ESBL) Detection a) Disc Susceptibility Test to Screen for ESBLs All isolates were screened for ESBL production using four indicator Cephalosporins, namely, Cefotaxime (30 $\mu \mathrm{g})$, Cefpodoxime $(10 \mu \mathrm{g})$, Ceftazidime $(30 \mu \mathrm{g})$, and one monobactam that is Aztreonam ( $30 \mu \mathrm{g})$. The isolates were considered to be resistant if the diameters of the inhibition zones are given below

\section{b) Confirmatory Test of ESBL by CLSI Disc Method}

For confirmation, the CLSI disc method, (9) Ceftazidime (30 $\mu \mathrm{g})$ discs were used, with and without Clavulanate (10 $\mu \mathrm{g})$.ESBL production was indicated by an increase in zone size of $5 \mathrm{~mm}$ or more in the disc with Ceftazidime and Clavulanic acid combination as compared to the disc of Ceftazidime alone. $(7,8,9)$

\section{RESULTS}

From October 2015 to January 2016, total 250 consecutive non-repetitive Enterobacteriaceae isolates were tested for detecting their antimicrobial susceptibility pattern and production of ESBLs by a screening and confirmatory testing.

\begin{tabular}{|c|c|}
\hline Organism $(n=250)$ & Total \\
\hline E.coli & 134 \\
\hline Klebsiella & 80 \\
\hline Enterobacter & 8 \\
\hline Proteus & 11 \\
\hline Citrobacter & 10 \\
\hline Serratia & 4 \\
\hline Morganella & 3 \\
\hline Total & 250 \\
\hline \multicolumn{2}{|c|}{ Table 1. Distribution of Organisms } \\
\hline
\end{tabular}

The source of the isolates was from urine, sputum, pus, blood, and CSF. The clinical spectrum represents out of 250 isolates, 145 are isolated from urine, 57 from pus, 45 from sputum, 2 from blood and 1 from CSF. The distribution pattern of isolated organisms shows that E.coli was the most commonly isolated organism followed by Klebsiella. Out of 250, 134 isolates were E.coli, after that Klebsiella, which were about 80 out of 250. Then Proteus (11), Citrobacter (10), Enterobacter (8), Serratia (4) and Morganella (3) respectively.

E.coli are predominantly isolated from urine samples, that is $81 \%(108 / 134)$. Then from pus that is $14(19 / 134)$ and from sputum, blood and CSF are $3 \%, 1 \%$, and $1 \%$ respectively.

\begin{tabular}{|c|c|c|}
\hline $\begin{array}{c}\text { Organism } \\
\text { (n=250) }\end{array}$ & $\begin{array}{c}\text { ESBL Screening } \\
\text { Test Positive } \\
(\mathbf{n = 5 4 )}\end{array}$ & $\begin{array}{c}\text { ESBL Confirmatory } \\
\text { Test Positive } \\
(\mathbf{n}=\mathbf{5 0})\end{array}$ \\
\hline E.coli & 41 & 37 \\
\hline Klebsiella & 11 & 11 \\
\hline Enterobacter & 1 & 1 \\
\hline Citrobacter & 1 & 1 \\
\hline Proteus & 0 & 0 \\
\hline Serratia & 0 & 0 \\
\hline Morganella & 0 & 0 \\
\hline \multicolumn{2}{|c|}{ Table 2. Distribution of ESBLs on Enterobacteriaceae } \\
\hline
\end{tabular}

This study showed a moderate rate of circulating ESBLs producers in this hospital set up. Among the 250 tested strains of Enterobacteriaceae, 20\% (50/250) are ESBLs producer. Of which, E.coli is the most common ESBL producer, that is $74 \%(37 / 50)$. Followed by Klebsiella, 22\% $(11 / 50)$ and Enterobacter and Citrobacter are only $2 \%$. From the ESBL Screening tests, 54 organisms gave a positive screening test, but on confirmatory testing using Ceftazidime and Ceftazidime + clavulanic acid combination, only 50 isolates are detected as ESBL producer.

\begin{tabular}{|c|c|c|}
\hline Antibiotics (N=100) & Resistant (\%) & Sensitive (\%) \\
\hline Ampicillin & 86 & 14 \\
\hline Piperacillin & 80 & 20 \\
\hline Amoxiclav & 70 & 30 \\
\hline Cephalexin & 69 & 31 \\
\hline Cefuroxime & 60 & 40 \\
\hline Cefotaxime & 52 & 48 \\
\hline Ceftazidime & 52 & 48 \\
\hline Cefepime & 36 & 64 \\
\hline Gentamicin & 32 & 67 \\
\hline Netilmicin & 15 & 85 \\
\hline Amikacin & 13 & 87 \\
\hline Ciprofloxacin & 42 & 58 \\
\hline Norfloxacin & 38 & 62 \\
\hline Ofloxacin & 42 & 58 \\
\hline Nitrofurantoin & 26 & 74 \\
\hline Cotrimoxazole & 36 & 64 \\
\hline Tetracycline & 41 & 59 \\
\hline Cefoperazone/Sulbactam & 14 & 86 \\
\hline Piperacillin/Tazobactam & 12 & 88 \\
\hline Imipenem & 6 & 94 \\
\hline Meropenem & 6 & 94 \\
\hline Aztreonam & 41 & 59 \\
\hline Cefoxitin & 32 & 68 \\
\hline Colistin & 0 & 100 \\
\hline Table 3. Susceptibility Pattern of Enterobacteriaceae \\
\hline & & \\
\hline & & \\
\hline
\end{tabular}

Overall antibiotic resistance pattern of the isolates showed a high degree of resistance towards Ampicillin (86\%), Piperacillin (80\%), Amoxiclav (70\%) and third generation Cephalosporins (50\%). This was statistically significant with p-value $<0.001$. Imipenem (6\%), Meropenem (6\%), Colistin (0\%), Cefoperazone/Sulbactam (14\%), Piperacillin/ Tazobactam (10\%) and Am.

\section{DISCUSSION}

Drug resistance causes a therapeutic problem not only in the hospital settings but also in the community as most of the bacteria have acquired resistance to multiple antibiotics. 
Various drug resistance mechanisms of Enterobacteriaceae include extended spectrum $\beta$-lactamases (ESBL) production, AmpC $\beta$-lactamases production, efflux mechanism, plasmids, and porin deficiency. In clinical laboratory settings, commonly the commonly detected enzymes causing resistance is. Detection of ESBL from Enterobacteriaceae are important to improve the clinical management of patients suffering from infections and would also provide us with sound epidemiological data. However, laboratory methods for screening and confirmation of ESBL should be accurate, simple and rapid.

In the present study, out of 250 isolates studied, 134 (54\%) are E.coli, and are the predominant isolates, followed by Klebsiella spp which are about $80(32 \%)$ cases and 8 $(3.2 \%)$ cases of Enterobacter and 10 (4\%) cases of Citrobacter were obtained. This result is comparable with a similar study conducted by S. Polsfuss, G. V. Bloemberg et al, in their study, out of 2518 samples, 1435 (57\%) were E.coli, $459(18.2 \%)$ were Klebsiella, 178 (5.2\%) were Enterobacter and $1(0.1 \%)$ case of Citrobacter were isolated.(5) A study done by Tapan Majumdar, Shibabrata Bhattacharya et al,(10) found out of 200 tested strains of Enterobacteriaceae, 50 cases were reported as E.coli, 40 cases of Klebsiella spp, 12 cases of Enterobacter and 8 cases of Citrobacter were obtained. But in the study of Leslie Jose Selvaraj, Anoop Sinha et al, out of 993 Enterobacteriaceae Isolates, 510 cases were reported as E.coli, while 277 cases are Klebsiella spp. and 168 cases are Enterobacter spp.(11)

The present finding of antibiotic susceptibility pattern was out of 250 cases, antibiotic resistance was, Ampicillin $85.4 \%$, Cefuroxime $60.8 \%$, Ceftazidime 52\%, Cefepime $36.4 \%$, Cefoxitin $31.6 \%$ and Imipenem 5\% only. From the analysis, data obtained, based on the antibiotic resistance pattern, the drugs such as Ampicillin, Cefuroxime, Ceftazidime, Cefepime, and Imipenem shows a significant sensitivity pattern that is, $\mathrm{p}<.0001$

Similarly the Study of Tapan Majumdar, Shibabrata Bhattacharya et al, it was obtained that overall antibiotic resistant pattern of the isolates showed a high degree of resistance towards Amoxycillin, $3^{\text {rd }}$ generation Cephalosporins and Tetracycline whereas Cefepime, Imipenem Amino Glycosides were showing less resistance.(10) The study conducted by Anandkumar Harwalkar, Jagadeesh Sataraddi, Soham Gupta et al,(12) revealed that out of 153 isolates, (93.8\%) was resistant to Ampicillin, 62(38\%) were resistant to Trimethoprim/ Sulfamethoxazole and 61(37.4\%) were resistant to Gatifloxacin. Resistance to Tobramycin, Amoxicillin/Clavulanate, and Amikacin were noted in between $30 \%$ and $19.6 \%$ of isolates. Only 2 (1.2\%) Strains showed resistance to Imipenem. In the similar study conducted by Rong WANG, MM, Shangwei et al,(13) showed that $70.1 \%$ were resistant to Ampicillin, $44.2 \%$ were resistant to Cefuroxime, $31.2 \%$ were resistant to Ceftazidime, $20.8 \%$ were resistant to Cefepime, and $75.3 \%$ were resistant to Cefoxitin.

The isolates that producing Extended Spectrum Betalactamases are detected by using Double disc Synergy Test of CLSI, using Ceftazidime and Ceftazidime/clavulanic acid combination discs. In present work shows that, 37 cases of E.coli and 11 cases of Klebsiella produce Extended Spectrum $\beta$-lactamases (ESBL). The test data was performed to analyze the data, from this Statistical study, it is clear that Chi-square value to analyze the data, Chi-square $=267.64, \mathrm{p}<0.0001$ and Fischer's Exact statistic $=43.1, \mathrm{p}<0.03$. This implies that there exists a significant difference between the distribution of ESBLs on different Enterobacteriaceae.

In a similar study of Col Naveen Grover, Brig A. K. Sahnin, Col S Bhattacharya et al,(14) Their findings were out of 262 clinical isolate, 58 cases of E.coli, 43 cases of Klebsiella spp and 0 cases Proteus spp shows Extended Spectrum $\beta$ lactamase (ESBL) positive in Double disc Synergy Test for of CLSI. But in the study of S. Polsfuss, G.V. Bloemberg et al,(5) showed that, out of 2518 isolates, 94 cases of E.coli, 48 cases of Klebsiella and 2 cases of Proteus spp shows Extended Spectrum $\beta$ - lactamases.

The most frequently used antibiotics nowadays are the $\beta$ lactam antibiotics. $\beta$-lactamases production in an organism has been found to be on the rise leading to increased resistance towards $\beta$-lactam antibiotics. Extended Spectrum $\beta$-Lactamases (ESBL) and AmpC are the two primary $\beta$ lactamases producers that are an increasing cause of concern in hospitals as they produce a therapeutic confusion for the treating physician. In order to provide useful information for effective control and clinical therapy of infection, the resistance status and the rate of carrying ESBL Enterobacteriaceae were investigated by phenotypic methods.

\section{CONCLUSION}

Among all Enterobacteriaceae isolates, the most commonly isolated organism was E.coli followed by Klebsiella spp. Overall antibiotic resistance pattern of the isolates showed a high degree of resistance towards ampicillin, piperacillin, amoxiclav and third generation cephalosporins and fourth generation cephalosporins. Whereas imipenem, meropenem, colistin, cefoperazone/sulbactam, piperacillin/tazobactam and aminoglycosides were showing less resistance. In this hospital set up, 20\% (50/250) are ESBLs producers.

Characterization, identification of the antibiotic susceptibility patterns and detection of ESBL may aid in hospital infection control and help the physician to prescribe the most appropriate antibiotics.

\section{REFERENCES}

[1] EI-Hady SA, Adel LA. Adel Occurrence and detection of AmpC $\beta$-lactamases among Enterobacteriaceae isolates from patients at Ain Shams University Hospital. The Egyptian Journal of Medical Human Genetics 2015;16:239-44.

[2] Jacoby GA. AmpC $\beta$-lactamases. Clin Microbiol Rev 2009;22(1):161-82.

[3] Singer RS, Finch R, Wegener HC, et al. Antibiotic resistance-the interplay between antibiotic use in animals and human beings. The Lancet Infectious Diseases 2003;3(1):47-51.

[4] Chakraborty A, Adhikari P, Shenoy S, et al. Characterization of plasmid mediated AmpC producing E-coli clinical isolates from a tertiary care hospital in South India. Indian Journal of Pathology and Microbiology 2014;57(2):255-8. 
[5] Polsfuss S, Bloemberg GV, Giger J, et al. Evaluation of a diagnostic flow chart for detection and confirmation of extended spectrum b-lactamases (ESBL) in Enterobacteriaceae. Clinical Microbiology Infection 2012;18(12):1194-204.

[6] Wassef M Behiry I, Younam M, et al. Genotypic identification of AmpC $\beta$-lactamases production in Gram-negative Bacilli isolates. Jundishapur J Microbiol 2014;7(1):e8556.

[7] Gupta V. An update on newer $\beta$-lactamases. Indian Journal for Medical Research 2007;126(5):417-27.

[8] Winn WC, Koneman EW. Koneman's Color Atlas and Textbook of Diagnostic Microbiology. Lippincott Williams \& Wilkins 2006: p. 47. [cited 2014 Nov 18].

[9] CLSI. Performance Standards for Antimicrobial Susceptibility Testing. Approved Standards; Eighteenth Informational Supplement. M100-S18. Wayne, PA: Clinical and Laboratory Standards Institute, 2018.

[10] Majumdar T, Bhattacharya S, Bir R. Prevalence of extended spectrum $\quad \beta$-lactamases among enterobacteriaceae and pseudomonadaceae isolated at tertiary care set up in Tripura, India. Research \& Reviews: Journal of Microbiology and Biotechnology 2014;3(2):19-26.
[11] Selvaraj LJ, Sinha A. Occurance of extended spectrum and AmpC $\beta$-lactamases in a tertiary care Hospital in South Kerala. The Journal of The academy of Clinical Microbiologists 2008;10:61-9.

[12] Wang R, Wu S, Li X, et al. Detection of AmpC betalactamases and drug resistance of Enterobacter cloacae. Front Med China 2009;3(1):72-5.

[13] Harwalkar A, Sataraddi J, Gupta S, et al. The detection of ESBL-producing E.coli in patients with symptomatic urinary tract infections using different diffusion methods in a rural setting. Journal of Infection and Public Health 2013;6(2):108-14.

[14] Col Grover N, Sahni AK, Col Bhattacharya S. Therapeutic challenges of ESBLs and AmpC $\beta$ lactamases producers in a tertiary care center. Medical Journal Armed Fources India 2013;69(1):4-10. 